\title{
大叶藤黄醇的全合成研究
}

\author{
王学颖 ${ }^{-}$盘伊琳 ${ }^{\dagger}$ 郑昌武* 徐宏喜* \\ (上海中医药大学中药学院 上海 201203)
}

\begin{abstract}
摘要 多环多异戊烯基间苯三酚(PPAPs)类天然产物大叶藤黄醇(xanthochymol)具有良好的抗癌和抗菌活性，目前没有 任何关于其全合成的研究报道. 本研究以 3,3-二甲基戊二酸为起始原料, 通过一系列步骤制备线性链状底物, 再应用串 联 Dieckmann 缩合反应构建双环[3.3.1]壬烷-2,4,9-三酮核心骨架. 此方法成功地以 11 步反应实现了消旋体大叶藤黄醇 的全合成, 以 $10 \%$ 的总收率得到了目标产物. 通过本研究得到大叶藤黄醇侧链的一对非对映异构体, 在 ${ }^{1} \mathrm{H} \mathrm{NMR}^{\mathrm{N}}{ }^{13} \mathrm{C}$ NMR 谱图上明确了它们之间的区别, 也对未来分离鉴定和区分其侧链结构异构体提供了参考数据.
\end{abstract}

关键词 多环多异戊烯基间苯三酚(PPAPs); 双环 [3.3.1]壬烷; Dieckmann 缩合; 串联反应

\section{Studies toward the Total Synthesis of Xanthochymol}

\author{
Wang, Xueying ${ }^{\dagger} \quad$ Phang, Yee Lin ${ }^{\dagger} \quad$ Zheng, Changwu* $^{*} \quad$ Xu, Hongxi* \\ (School of Pharmacy, Shanghai University of Traditional Chinese Medicine, Shanghai 201203)
}

\begin{abstract}
Xanthochymol, a polycyclic polyprenylated acylphloroglucinol (PPAP) natural compound, has demonstrated good anticancer and antimicrobial properties. However, no related studies on the synthesis of xanthochymol have been reported hitherto. In this study, 3,3-dimethylglutaric acid was used as the starting material to synthesize a key linear precursor via several steps, followed by a domino Dieckmann condensation reaction to construct the bicyclo[3.3.1]nonane-2,4,9-trione core skeleton. With that, the total synthesis of $( \pm)$-xanthochymol in 11 steps with a total yield of $10 \%$ has been first accomplished. By obtaining the side chain diastereomers of xanthochymol through this study, we have clarified the difference between them on the ${ }^{1} \mathrm{H}$ NMR and ${ }^{13} \mathrm{C}$ NMR spectra while at the same time provided references for future isolation, identification, and discrimination of xanthochymol from its side chain structural isomers.

Keywords polycyclic polyprenylated acylphloroglucinols (PPAPs); bicyclo[3.3.1]nonanes; Dieckmann condensation; domino reaction
\end{abstract}

多环多异戊烯基间苯三酚(PPAPs)类化合物在抗肿 瘤、抗炎、抗艾滋病病毒(HIV)、抗菌和抗抑郁等方面 均展示良好的生物活性, 近年来也因此引起了药物化学 家的广泛关注 ${ }^{[1-4]}$. 自 1971 年在金丝桃属植物中提取分 离出第一个 PPAPs 类化合物贯叶金丝桃素(hyperforin) 以来 ${ }^{[5]}$, 目前为止已有超过 900 个 PPAPs 类化合物从金 丝桃科和藤黄科植物中被发现 ${ }^{[6]}$. 大部分的 PPAPs 类化 合物具有一个较为罕见的双环[3.3.1]壬烷-2,4,9-三酮结 构, 根据环上取代酰基位置的不同又可分为 $\mathrm{A}$ 型和 $\mathrm{B}$ 型 PPAPs 类化合物: A 型的酰基在 C- 1 位, 而 B 型的酰基在 C-3 位. 早期有许多课题组对 PPAPs 类化合物进行了深 入的合成研究 ${ }^{[7-11]}$, 但直到 2005 年, 第一个天然 PPAPs 类化合物 garsubellin A 才被 Shibasaki 课题组 ${ }^{[12]}$ 成功合
成. 近年来, PPAPs 类天然产物已经成为全合成领域比 较热门的研究方向. 进一步分析已有 PPAPs 类天然产物 的全合成报道, 发现这些研究主要集中于侧链不含手性 或者侧链与 4-位羰基形成氧杂六元环的 PPAPs 类天然 产物, 而含环外手性中心的 PPAPs 类天然产物的合成研 究极少被报道.

含环外手性侧链的一个重点 PPAPs 分子是大叶藤 黄醇(1, xanthochymol)(图 1). 这个化合物在 1973 年从大 叶藤黄(Garcinia xanthochymus) 中被发现和报道 ${ }^{[13]}$, 后 来也在版纳藤黄、菲岛福木等藤黄属植物中被分离提取. 研究表明, 大叶藤黄醇具有显著的抗癌和抗菌等作用. 它可以有效抑制结肠癌细胞的生长 ${ }^{[14-15]}$, 对白血病细胞 系也有良好的细胞调亡诱导作用 ${ }^{[16]}$. 大叶藤黄醇对抗

* Corresponding authors. E-mail: zhengcw@shutcm.edu.cn; xuhongxi88@gmail.com Received June 3, 2021; revised July 8, 2021; published online August 9, 2021.

Project supported by the National Natural Science Foundation of China (No. 81973438). 国家自然科学基金(No. 81973438)资助项目. 
耐药性金黄色葡萄球菌 (Methicillin-resistant Staphylococcus aureus, MRSA)的最小抑菌浓度(MIC)在 3.13 $12.50 \mu \mathrm{g} / \mathrm{mL}$ 之间, 与临床常用药万古霉素有相当的抗 菌作用 ${ }^{[17]}$. 然而由于其 5-位侧链上含有一个极易发生异 构化的双键, 使分离得到的大叶藤黄醇往往混有末端双 键异构体, 如 guttiferone E (2), cycloxanthochymol (3, 环 大叶藤黄醇)和 isoxanthochymol (4, 异大叶藤黄醇)等 (图 1). 尽管目前化合物 $\mathbf{1 , 2}$ 和 $\mathbf{4}$ 的绝对构型和化合物 $\mathbf{3}$ 的相对构型已知，但这些异构体极性类似，难以采用一 般的分离手段将它们分离提纯 ${ }^{[18-19]}$. 因此, 设计出一种 快速简便的合成方法制备此类天然产物, 对后续研究它 的药用价值有着重要的意义. 同时需要注意的是, 由于 大叶藤黄醇的 5-位侧链上含有立体化学中心, 为它的合 成也带来了挑战.

根据逆合成分析 (Scheme 1), 断开大叶藤黄醇(1) C-3 位上的酰基可得到双环 [3.3.1]壬烷-2,4,9-三酮(5)的 结构, 再将其逆向分解得到多取代的线性前体 6 . 根据
我们之前发展的方法 ${ }^{[20-21]}$, 大叶藤黄醇的 C-3-C-4 和 C-1-C-9 化学键可通过中间体 6 的串联 Dieckmann 反 应来构建，而 C-5 位的侧链则可以通过中间体 7 的烷基 化反应得到. 利用这个合成策略, C-5 位的手性侧链可 以被模块化地变换，简便地实现各种带有不同立体中心 支链结构的 B 型 PPAPs 类化合物的合成. 最后，作为关 键前体的多取代链状底物 7, 可以由廉价易得的 3,3-二 甲基戊二酸 $(8)$ 轻松地与异戊烯基溴(9)发生烷基化反应, 接着与丙二酸二甲酯(10)发生亲核取代反应来获得.

\section{1 结果与讨论}

基于以上设想，我们决定沿用本课题组之前发展的

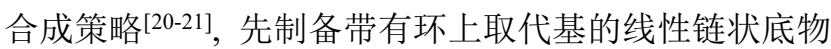
11, 然后利用三甲基铝、乙硫醇原位形成的路易斯酸 $\mathrm{Me}_{2} \mathrm{AlSEt}$ 催化串联 Dieckmann 反应一步成环(Eq. 1). 根 据之前的机理分析，此方法具有高度的非对映选择性， 合成的双环[3.3.1]壬烷骨架为内型，尚未分离出其外型

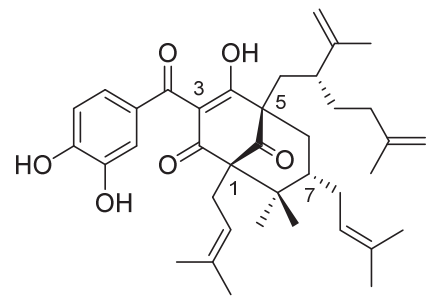

xanthochymol (1)

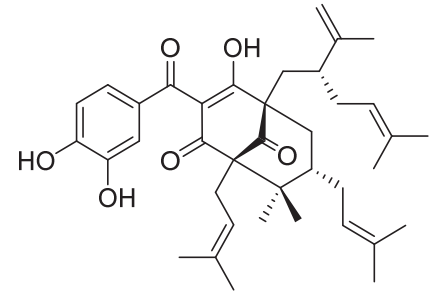

guttiferone $\mathrm{E}(2)$

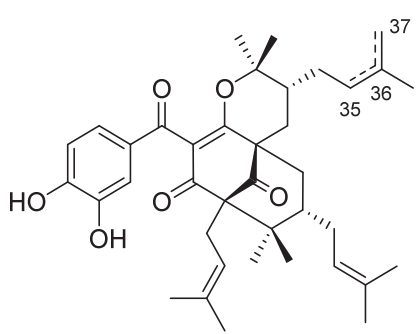

$\Delta^{36}$ cycloxanthochymol (3) $\Delta^{35}$ isoxanthochymol (4)

图 1 大叶藤黄醇及其异构体

Figure 1 Xanthochymol and its isomers
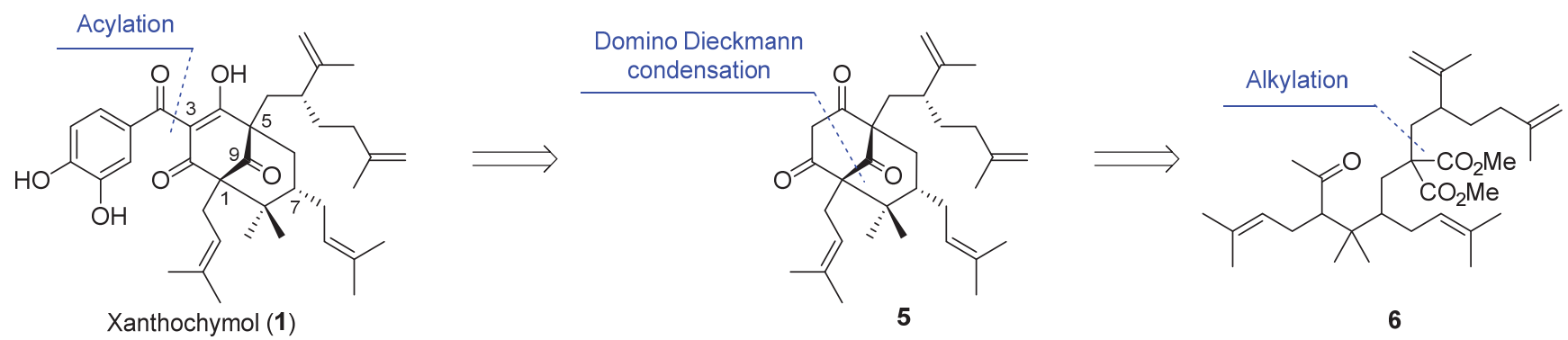

6
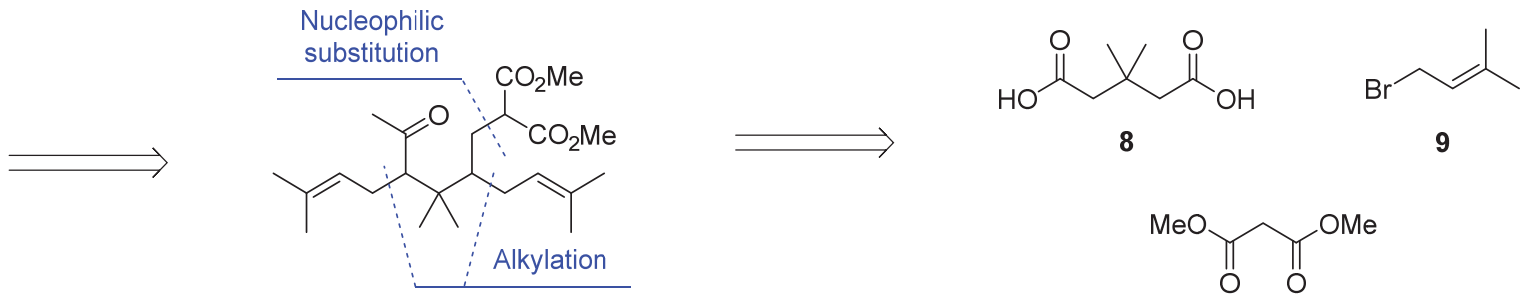

7<smiles>COC(=O)CC(=O)OC</smiles>

图式 1 大叶藤黄醇的逆合成分析

Scheme 1 Retrosynthetic analysis of xanthochymol 
异构体.

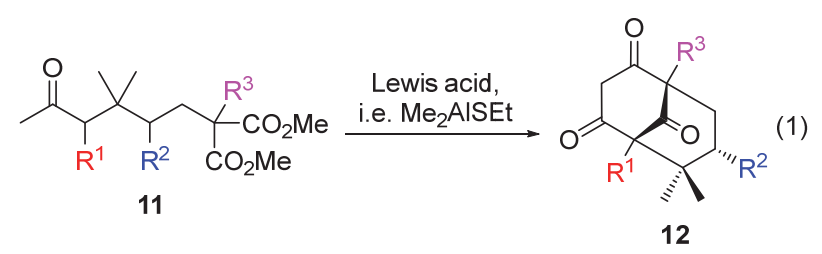

合成策略以 3,3-二甲基戊二酸 (8) 为起始原料, 经 5 步已知反应得到半缩醛 $\mathbf{1 3}^{[20]}$, 但半缩醛 13 不稳定, 在 酸、碱或加热条件下容易脱水生成烯醚 17 (Scheme 2). 我们尝试通过开环卤代反应得到化合物 15 或类似的氯 代和碘代产物, 但 13 在多种卤化条件如 $\mathrm{I}_{2} / \mathrm{PPh}_{3} /$ 咪唑, $\mathrm{NIS} / \mathrm{PPh}_{3}, \mathrm{NBS} / \mathrm{PPh}_{3}, \mathrm{NaI} /\left(\mathrm{CH}_{3}\right)_{3} \mathrm{SiCl}, \mathrm{PPh}_{3} \cdot \mathrm{Br}_{2} /$ 吡啶, $\mathrm{PPh}_{3} / \mathrm{CBr}_{4}$ 和 $\mathrm{PPh}_{3} / \mathrm{CCl}_{4}$ 等条件下, 除了脱水产物 17 以外 没有目标产物产生. 只有在 $N$-氯代丁二酰亚胺(NCS)、 三苯基膦和碳酸钾的条件下有不到 $30 \%$ 的氯代产物出 现. 进一步优化条件时发现, 在使用 $\mathrm{PPh}_{3} \cdot \mathrm{Br}_{2}$ /吡啶的条 件时, 所使用的 $\mathrm{PPh}_{3} \cdot \mathrm{Br}_{2}$ 是购买的试剂, 产物只有脱水 产物 17 生成. 考虑到 $\mathrm{PPh}_{3} \cdot \mathrm{Br}_{2}$ 是这个反应的核心试剂, 我们尝试在三澳化吡啶/三苯基膦的条件下原位制备 $\mathrm{PPh}_{3} \cdot \mathrm{Br}_{2}$ 试剂(Scheme 3), 期望这一操作可以提高该试 剂的活性, 从而使反应成功. 最终反应在加热条件下成 功得到溴代产物 $15^{[21]}$. 进一步研究发现当底物 13 与 $\mathrm{PPh}_{3} \cdot \mathrm{Br}_{2}$ 反应时, 会发生竞争反应(Scheme 2 ). 常温时反 应只得到 17 , 没有产物 15 生成, 当升高温度回流时产
物 15 的产率有 $90 \%$. 最初假设的升高温度会导致脱水 产物增多并没有出现，表明温度在这个反应里起到至关 重要的作用.

随后，产物 15 在碱 $\mathrm{NaH}$ 的条件下以 $\mathrm{N}, \mathrm{N}$-二甲基甲 酰胺(DMF)为溶剂, 与丙二酸二甲酯(10)发生烷基化反 应得到链状底物 7 (Scheme 3) ) $^{[21]} .7$ 有一对顺式和反式的 非对映异构体，可用制备液相柱分开，但由于这种立体 选择性的差别在环化过程中会被消除，因此在实验过程 中这对非对映异构体不被分开，直接进行下一步反应. 接下来, 化合物 7 与制备的侧链 $18^{[22]}$ 再经一步烷基化反 应, 生成最终的链状成环前体 $\mathbf{6}$. 由于化合物 6 含有 3 个立体中心，因此可能生成 4 对外消旋体. 得到的产物 通过柱层析分离得到两组混合产物, 通过核磁分析也得 到了确认. 最后 6 在乙硫醇、三甲基铝催化下发生一步 串联 Dieckmann 成环反应. 在这个过程中, 反应开始检 测到 8 元环产物生成, 反应结束后 8 元环产物消失, 说 明在乙硫醇、三甲基铝的条件下，反应先是形成 8 元环， 再二次成环生成内型的 [3.3.1]桥环 ${ }^{[23]}$. 根据之前的机理 分析 ${ }^{[20]}$, 起始原料 $\mathbf{6}$ 中存在的多个异构体对产物 $\mathbf{5}$ 的构 型没有影响，原因为: (1)酮羰基邻位的立体中心构型在 反应中烯醇化而消除; (2)串联成环反应由于位阻效应只 生成内型的[3.3.1]环状结构. 因此该反应仅得到一对因 侧链立体中心影响而生成的非对映异构体 $( \pm)-5 \mathbf{a}$ 和 $( \pm)-\mathbf{5 b}$, 比值为 $1: 1$.

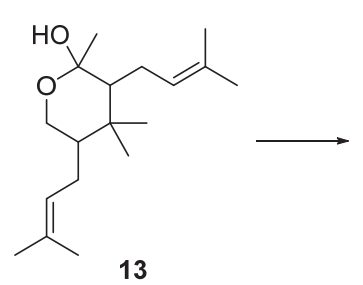

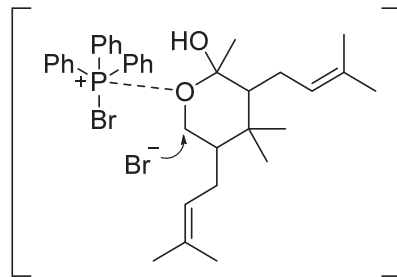

14
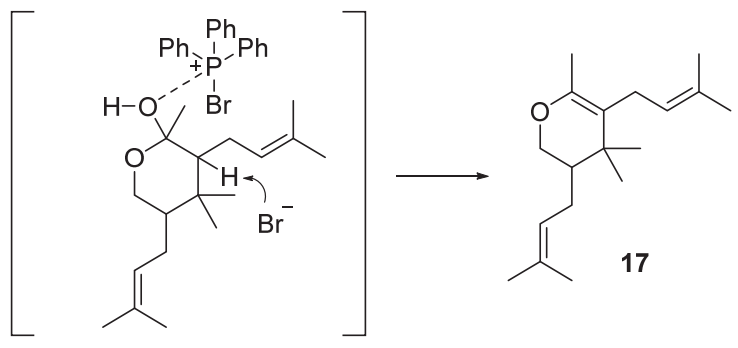

16

图式 2 化合物 $\mathbf{1 3}$ 的开环竞争反应

Scheme 2 Competitive ring-opening reaction of compound 13 
<smiles>CC(C)=CCC1COC(C)(O)C(CC=C(C)C)C1(C)C</smiles>

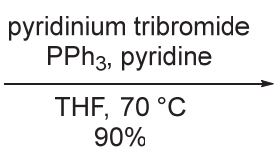

$90 \%$

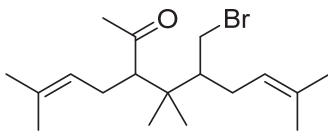

15

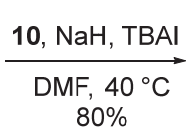
$80 \%$<smiles>CC(=O)CC(CC(CC=C(C)C)C(C)=O)C(C)(C)C(CC=C(C)C)C(C)=O</smiles>

7
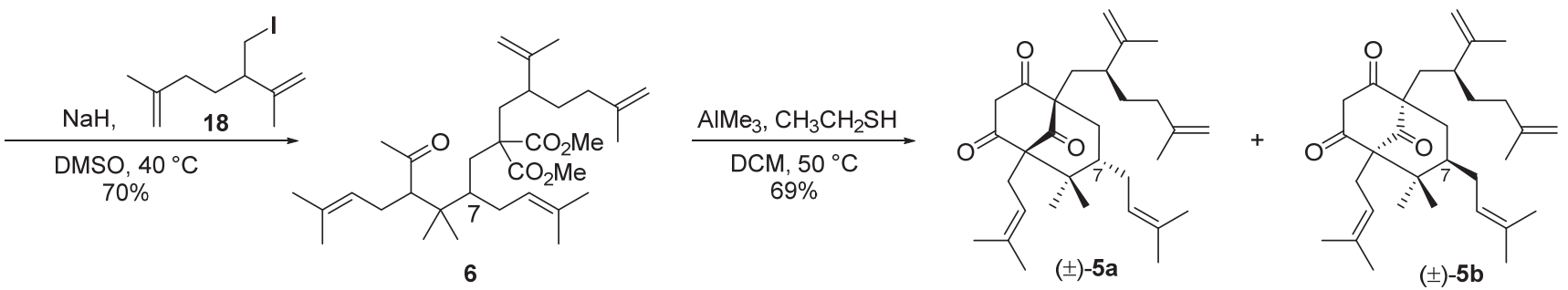

图式 3 双环[3.3.1]壬烷的构建

Scheme 3 Synthesis of bicyclo[3.3.1]nonane ring

在完成双环[3.3.1]壬烷-2,4,9-三酮骨架的建立之后, 我们对 C-3 位的酰基化反应进行了考察, 发现叔丁醇钾 或三乙胺条件下都不能顺利发生 $C$-酰基化反应, 即便 增加叔丁醇钾和酰氰的用量或是升高温度时, 反应现象 均不是很佳, 仅能检测到微量的产物. 此后尝试了大多 数的有机碱, 如 $\mathrm{NaH}$ 、六甲基二硅基胺基锂(LHMDS)、 二异丙基氨基锂(LDA)、 $n-\mathrm{BuLi}$ 等都没有发生反应, 而 当反应的酰基化试剂从制备的 19 换成有商品原料的 99\%纯的苯甲酰氧时，很容易得到了酰基化的产物. 这 提示原料底物中或许有种杂质可对反应产生影响, 因此 我们将 19 的纯度从 95\%提高到了 $99 \%$ 以上, 成功得到 酰基化的产物. 最终对化合物 $\mathbf{5 a}$ 和 $\mathbf{5 b}$ 用三氯化矧催化、 三乙胺为碱、甲苯做溶剂、与带保护基的苯甲酰氰(19)
完成酰基化反应(Scheme 4). 此反应对酸极为敏感, 即 使反应体系仅含有少量弱酸也不会有产物生成, 再加上 带保护基的苯甲酰氰不易纯化且容易变质, 使苯甲酰氰 部分变成苯甲酸, 因此这一合成步骤有一定的难度. 最 终酰基化产物再经碳酸钾水解，以 $51 \% \sim 55 \%$ 的产率 (两步反应)生成产物 $\mathbf{1}$ 或者 $\mathbf{1}$, 经过氢谱和碳谱核对(见 辅助材料), 确认合成了消旋体的天然产物大叶藤黄醇 $\mathbf{1}^{[19]}$.

\section{2 结论}

以 3,3-二甲基戊二酸为原料, 通过一系列步骤制备 了带有 C-1、C-5 和 C-7 侧链的关键多取代线性前体, 而 后在三甲基铝、乙硫醇原位生成的路易斯酸 $\mathrm{Me}_{2} \mathrm{AlSEt}$

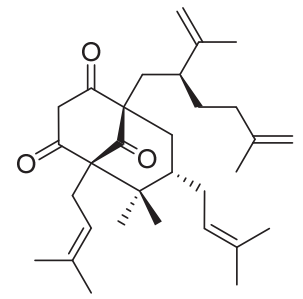

( \pm -5a

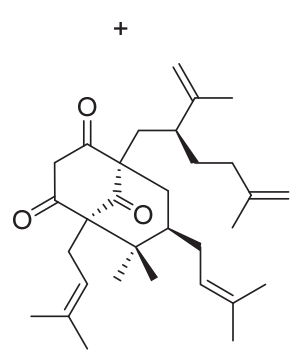

$( \pm)-5 b$

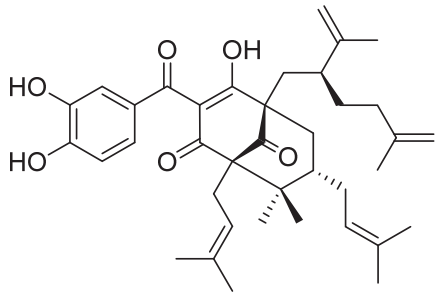

$( \pm)-1^{\prime}$
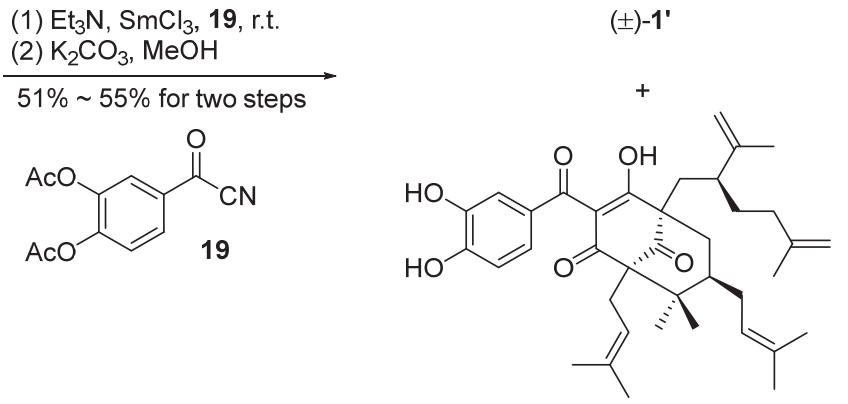

( \pm )-Xanthochymol (1)

图式 4 大叶藤黄醇的合成

Scheme 4 Synthesis of xanthochymol 
促进下，通过串联 Dieckmann 缩合的策略一步制备外消 旋的天然产物大叶藤黄醇. 合成中, 我们探讨了 $\mathrm{PPh}_{3} \cdot \mathrm{Br}_{2}$ 试剂的活性对溴化反应的影响, 以及试剂的纯 度对酰基化反应的影响等, 为该两类反应在天然产物合 成中的应用提供了有益的参考. 通过变换线性前体的 C-1、C-5 和 C-7 侧链, 我们相信这个方法也适用于其他 PPAPs 类天然产物的合成, 可进一步研究和拓展这个反 应的普适性。

\section{3 实验部分}

\section{1 仪器与试剂}

本研究所使用的反应试剂和无水溶剂均从泰坦科 技购买, 无特殊说明实验均在无水无氧条件下操作. 实 验操作过程中使用薄层色谱(TLC)监测反应进程, 监测 方式包括: 紫外灯苂光显示、茴香醛、碱性高锰酸钾与 磷钿酸显色. 核磁共振(NMR) 氢谱、碳谱及二维谱由 AV-400 MHz(德国 Bruker 公司), AV-600 MHz(德国 Bruker 公司)或 Agilent $400 \mathrm{MHz}$ (美国 Agilent 公司)核磁 共振仪测定, 以 $\mathrm{CDCl}_{3}, \mathrm{CD}_{3} \mathrm{OD}$ 或 DMSO- $d_{6}$ 溶剂峰为基 准. ${ }^{1} \mathrm{H}$ NMR 中残余溶剂峰位置 $(\delta)$ 如下: $\mathrm{CDCl}_{3}$ 为 7.26, $\mathrm{CD}_{3} \mathrm{OD}$ 为 $3.31, \mathrm{DMSO}-d_{6}$ 为 2.50 ; 而 ${ }^{13} \mathrm{C} \mathrm{NMR}$ 中残留溶 剂峰位置 $(\delta): \mathrm{CDCl}_{3}$ 为 77.2, $\mathrm{CD}_{3} \mathrm{OD}$ 为 $49.0, \mathrm{DMSO}-d_{6}$ 为 39.5. 红外光谱(IR)由 Perkin- Elmer Spectrum 983G 光 谱仪(美国 Perkin-Elmer 公司)测定. 高分辨质谱由 SYNAPT G2-Si HDMS 型质谱仪(美国 Waters 公司)测定. 高效液相色谱(HPLC)由美国 Waters 公司 2535 系列仪器 测定, 所用分析柱为 Xbridge $\mathrm{C} 18$ 柱 $(4.6 \mathrm{~mm} \times 250 \mathrm{~mm}$, $5 \mu \mathrm{m}$ )(美国 Waters 公司), 样品制备分离所用制备柱为 Xbridge Prep C18 OBD 制备柱 $(19 \mathrm{~mm} \times 250 \mathrm{~mm}, 5$ $\mu \mathrm{m})($ 美国 Waters 公司)拌样使用. 硅胶为山东烟台江友 硅胶开发有限公司生产的 100 200 目硅胶; 柱层析使 用硅胶为烟台江友硅胶开发有限公司生产的 $300 \sim 400$ 目硅胶. 旋光由 Autopol 型自动波长半导体控温旋光仪 (美国 Rudolph 公司)测定.

\section{2 化合物 6 的合成}

将混合物 7 (7.6 g, $19.3 \mathrm{mmol}$ )溶于 $150 \mathrm{~mL}$ 二甲基亚 砜(DMSO), 并将反应体系于冰水浴剧烈搅拌, 分批缓 慢加入 $\mathrm{NaH}(60 \%$ in mineral oil, $1.2 \mathrm{~g}, 29 \mathrm{mmol})$. 加料结 束后, 将混合物体系升温至 $40{ }^{\circ} \mathrm{C}$ 并继续摚拌 $0.5 \mathrm{~h}$. 接 着将反应冷却至室温后, 滴加化合物 18 (11 g, 42 $\mathrm{mmol})$, 并在室温继续摚拌 $6 \mathrm{~h}$. TLC 监测到反应结束后, 加入 $100 \mathrm{~mL} \mathrm{H}_{2} \mathrm{O}$ 淬灭反应, 并用乙酸乙酯萃取 $(150 \mathrm{~mL}$ $\times 3$ ). 合并有机相, 用饱和食盐水洗涤有机相, 无水 $\mathrm{Na}_{2} \mathrm{SO}_{4}$ 干燥, 减压浓缩除去有机溶剂, 柱层析分离 $\left(R_{\mathrm{f}}=0.5\right.$, 石油醚/乙酸乙酯, $\left.V: V=10: 1\right)$ 纯化得到 7.3 $\mathrm{g}$ 淡黄色油状液体化合物 6, 产率 70\%.

2-(4-乙酰基-3,3,7-三甲基-2,4-二异戊烯基丁 基)-2-(2-异丙烯基-6-甲基已烯基)丙二酸二甲酯(6a)(多 个异构体): ${ }^{1} \mathrm{H}$ NMR (400 MHz, $\left.\mathrm{CDCl}_{3}\right) \delta: 5.14 \sim 4.94$ (m, $2 \mathrm{H}), 4.90 \sim 4.52(\mathrm{~m}, 4 \mathrm{H}), 3.75 \sim 3.59(\mathrm{~m}, 6 \mathrm{H}), 2.94 \sim 2.78$ (m, $1 \mathrm{H}), 2.44 \sim 2.13(\mathrm{~m}, 3 \mathrm{H}), 2.10 \sim 2.06(\mathrm{~m}, 3 \mathrm{H}), 2.05 \sim$ $1.88(\mathrm{~m}, 3 \mathrm{H}), 1.88 \sim 1.74(\mathrm{~m}, 4 \mathrm{H}), 1.71 \sim 1.67(\mathrm{~m}, 3 \mathrm{H})$, $1.66 \sim 1.59(\mathrm{~m}, 9 \mathrm{H}), 1.59 \sim 1.52(\mathrm{~m}, 7 \mathrm{H}), 1.51 \sim 1.43(\mathrm{~m}$, $1 \mathrm{H}), 1.42 \sim 1.34(\mathrm{~m}, 2 \mathrm{H}), 0.90 \sim 0.82(\mathrm{~m}, 6 \mathrm{H}) ;{ }^{13} \mathrm{C} \mathrm{NMR}$ $\left(151 \mathrm{MHz}, \mathrm{CDCl}_{3}\right) \delta: 213.4,213.3,172.6,172.5,172.3$, $172.0,146.1,145.8,145.7,145.5,133.1,133.1,130.1$, $129.9,125.2,125.0,122.4,122.4,113.3,113.2,109.7$, $109.6,56.9,56.8,56.5,56.4,52.2,52.0,52.0,51.6,42.9$, $42.5,40.6,40.3,40.1,40.1,37.6,37.3,35.5,35.2,35.2$, $35.0,34.5,34.4,33.0,32.4,28.9,28.2,27.2,27.1,25.8$, $25.7,25.7,22.6,22.6,22.2,22.0,21.5,21.5,18.1,18.0$, 17.8, 17.7, 17.5. HRESIMS calcd for $\mathrm{C}_{33} \mathrm{H}_{55} \mathrm{O}_{5}[\mathrm{M}+\mathrm{H}]^{+}$ 531.4049 , found 531.4069 .

2-(4-乙酰基-3,3,7-三甲基-2,4-二异戊烯基丁 基)-2-(2-异丙烯基-6-甲基己烯基)丙二酸二甲酯(6b)(多 个异构体): ${ }^{1} \mathrm{H}$ NMR (400 MHz, $\left.\mathrm{CDCl}_{3}\right) \delta: 5.23 \sim 4.51$ (m, $6 \mathrm{H}), 3.78 \sim 3.69(\mathrm{~m}, 3 \mathrm{H}), 3.65 \sim 3.58(\mathrm{~m}, 3 \mathrm{H}), 2.84 \sim 2.47$ $(\mathrm{m}, 2 \mathrm{H}), 2.42 \sim 2.11(\mathrm{~m}, 4 \mathrm{H}), 2.08 \sim 2.03(\mathrm{~m}, 3 \mathrm{H}), 1.99 \sim$ $1.71(\mathrm{~m}, 5 \mathrm{H}), 1.70 \sim 1.54(\mathrm{~m}, 20 \mathrm{H}), 1.52 \sim 1.37(\mathrm{~m}, 2 \mathrm{H})$, $0.98 \sim 0.80(\mathrm{~m}, 6 \mathrm{H}) ;{ }^{13} \mathrm{C}$ NMR $\left(151 \mathrm{MHz}, \mathrm{CDCl}_{3}\right) \delta$ : $213.8,213.7,213.3,213.3,172.8,172.5,172.0,172.0$, $169.7,169.7,169.4,169.4,145.6,145.6,145.5,145.5$, $145.3,145.3,145.0,145.0,132.9,132.9,132.9,132.8$, $130.2,130.0,129.9,125.2,124.8,124.8,124.7,122.4$, $122.3,122.0,121.9,113.5,113.5,113.3,113.3,113.3$, $110.0,110.0,109.9,109.8,60.9,60.9,56.9,56.9,56.5$, 56.3, 56.0, 52.9, 52.8, 52.1, 52.1, 51.8, 51.6, 42.3, 42.3, $40.2,39.9,39.9,39.8,36.4,36.0,35.9,35.8,35.4,35.3$, $35.3,35.2,34.6,34.6,34.1,34.1,33.4,33.1,32.1,31.4$, $30.7,30.7,30.2,30.1,28.8,28.8,26.3,26.2,25.9,25.9$, $25.8,25.8,25.7,22.6,22.5,22.5,22.5,21.5,21.4,21.3$, $20.9,18.1,18.1,18.1,18.0,17.8,17.8,17.7,17.7,17.4$, 17.3. HRESIMS calcd for $\mathrm{C}_{33} \mathrm{H}_{55} \mathrm{O}_{5}[\mathrm{M}+\mathrm{H}]^{+} 531.4049$, found 531.4053 .

\section{3 化合物 $\mathbf{5}$ 的合成}

在室温及 $\mathrm{N}_{2}$ 氛围保护下, 将乙硫醇 $(97 \%, 1.22 \mathrm{~mL}$, $17 \mathrm{mmol}$ )溶解在 $12 \mathrm{~mL} \mathrm{CH} \mathrm{Cl}_{2}$ 中. 缓慢滴加三甲基铝 $(1.0 \mathrm{~mol} / \mathrm{L}$ 庚烷溶液, $17 \mathrm{~mL}, 17 \mathrm{mmol})$, 滴加过程中小 心, 反应会剧烈放出气体. 滴加完毕后, 将反应体系在 该温度下继续摚拌 $0.5 \mathrm{~h}$. 减压除去体系中 $\mathrm{CH}_{2} \mathrm{Cl}_{2}$ 和庚 
烷, 将化合物 6 (600 mg, $1.13 \mathrm{mmol}$ )溶于 $12 \mathrm{~mL} \mathrm{CH}_{2} \mathrm{Cl}_{2}$ 中, 升温至 $50{ }^{\circ} \mathrm{C}$ 搅拌 $12 \mathrm{~h}$, 降至室温并慢慢滴加 $\mathrm{H}_{2} \mathrm{O}$ 淬灭反应. 随后滴加 $1 \mathrm{~mol} / \mathrm{L} \mathrm{HCl}$ 溶液至白色乳浊液变 澄清, 室温继续摚拌 $10 \mathrm{~min}$. 用 $\mathrm{CH}_{2} \mathrm{Cl}_{2}$ 萃取水相 $(20$ $\mathrm{mL} \times 3)$, 合并有机相, 饱和氯化钠溶液洗涤有机相, 并 用无水 $\mathrm{Na}_{2} \mathrm{SO}_{4}$ 干燥. 减压浓缩后通过柱层析分离纯化 $\left(R_{\mathrm{f}}=0.15\right.$, 石油醚/乙酸乙酯, $V: V=10 ： 1$ )得到 $300 \mathrm{mg}$ 呈黄色油状液体的双环 [3.3.1]壬烷-2,4,9-三酮产物 $\mathbf{5 a}$ 和 5b, $d r$ 值为 $1: 1$, 产率 $69 \%$.

(1R,5S,7R)-6,6-二甲基-1-(5-甲基-2-异丙烯基-5-己 烯)-5,7-二异戊烯基-2,4,9-三酮-二环[3.3.1]壬烷(5a): ${ }^{1} \mathrm{H}$ NMR $\left(600 \mathrm{MHz}, \mathrm{CDCl}_{3}\right) \delta: 4.93 \sim 4.88(\mathrm{~m}, 1 \mathrm{H}), 4.86 \sim$ $4.79(\mathrm{~m}, 1 \mathrm{H}), 4.71 \sim 4.63(\mathrm{~m}, 2 \mathrm{H}), 4.60 \sim 4.52(\mathrm{~m}, 2 \mathrm{H})$, 3.54 (d, $J=17.1 \mathrm{~Hz}, 1 \mathrm{H}), 3.45$ (d, $J=17.1 \mathrm{~Hz}, 1 \mathrm{H}), 2.82$ (tt, $J=10.8,4.2 \mathrm{~Hz}, 1 \mathrm{H}), 2.59 \sim 2.53(\mathrm{~m}, 2 \mathrm{H}), 2.29(\mathrm{dd}$, $J=13.9,1.4 \mathrm{~Hz}, 1 \mathrm{H}), 2.13(\mathrm{dd}, J=21.7,7.5 \mathrm{~Hz}, 1 \mathrm{H})$, $2.10 \sim 2.03(\mathrm{~m}, 1 \mathrm{H}), 1.96(\mathrm{dd}, J=13.9,6.3 \mathrm{~Hz}, 1 \mathrm{H}), 1.86$ (td, $J=7.5,5.6,2.9 \mathrm{~Hz}, 2 \mathrm{H}), 1.71(\mathrm{~s}, 3 \mathrm{H}), 1.70 \sim 1.67(\mathrm{~m}$, $1 \mathrm{H}), 1.65(\mathrm{~s}, 3 \mathrm{H}), 1.64 \sim 1.62(\mathrm{~m}, 3 \mathrm{H}), 1.62 \sim 1.60(\mathrm{~m}$, $3 \mathrm{H}), 1.48(\mathrm{~d}, J=19.1 \mathrm{~Hz}, 6 \mathrm{H}), 1.39$ (ddt, $J=20.5,10.8$, $6.3 \mathrm{~Hz}, 3 \mathrm{H}), 1.31 \sim 1.27(\mathrm{~m}, 1 \mathrm{H}), 1.24(\mathrm{~s}, 3 \mathrm{H}), 0.97$ (s, $3 \mathrm{H}) ;{ }^{13} \mathrm{C}$ NMR (151 MHz, $\left.\mathrm{CDCl}_{3}\right) \delta: 210.9,203.0,202.7$, $148.5,145.9,136.2,133.6,122.5,117.9,114.3,109.6$, 71.0, 63.6, 62.9, 52.3, 46.7, 44.7, 43.2, 38.3, 35.3, 32.1, 29.0, 26.9, 26.4, 26.0, 25.8, 23.3, 22.6, 18.0, 17.9, 16.5 . HRESIMS calcd for $\mathrm{C}_{31} \mathrm{H}_{47} \mathrm{O}_{3}[\mathrm{M}+\mathrm{H}]^{+} 467.3525$, found 467.3550 .

(1 $R, 5 S, 7 R$ )- 6,6 -二甲基-1-(5-甲基-2-异丙烯基-5-己 烯)-5,7-二异戊烯基-2,4,9-三酮-二环[3.3.1]壬烷(5b): ${ }^{1} \mathrm{H}$ NMR (600 MHz, $\left.\mathrm{CDCl}_{3}\right) \delta: 4.85$ (q, $\left.J=7.8,7.3 \mathrm{~Hz}, 2 \mathrm{H}\right)$, $4.69(\mathrm{t}, J=1.8 \mathrm{~Hz}, 1 \mathrm{H}), 4.64(\mathrm{~s}, 1 \mathrm{H}), 4.63 \sim 4.62(\mathrm{~m}, 1 \mathrm{H})$, $4.44(\mathrm{~d}, J=2.7 \mathrm{~Hz}, 1 \mathrm{H}), 3.67$ (d, $J=16.9 \mathrm{~Hz}, 1 \mathrm{H}), 3.34$ (d, $J=16.9 \mathrm{~Hz}, 1 \mathrm{H}), 2.56(\mathrm{t}, J=6.4 \mathrm{~Hz}, 2 \mathrm{H}), 2.35 \sim 2.26(\mathrm{~m}$, $2 \mathrm{H}), 2.22 \sim 2.13(\mathrm{~m}, 2 \mathrm{H}), 2.04(\mathrm{dd}, J=13.9,6.3 \mathrm{~Hz}, 1 \mathrm{H})$, $1.83(\mathrm{td}, J=8.8,6.0 \mathrm{~Hz}, 2 \mathrm{H}), 1.77$ (s, 3H), $1.71(\mathrm{~s}, 3 \mathrm{H})$, $1.69(\mathrm{~s}, 3 \mathrm{H}), 1.63(\mathrm{~s}, 3 \mathrm{H}), 1.62(\mathrm{~s}, 3 \mathrm{H}), 1.60 \sim 1.58(\mathrm{~m}$, $1 \mathrm{H}), 1.54(\mathrm{~s}, 3 \mathrm{H}), 1.48 \sim 1.40(\mathrm{~m}, 3 \mathrm{H}), 1.34 \sim 1.30(\mathrm{~m}$, 1H), $1.26(\mathrm{~s}, 3 \mathrm{H}), 0.96(\mathrm{~s}, 3 \mathrm{H}) ;{ }^{13} \mathrm{C}$ NMR (151 MHz, $\left.\mathrm{CDCl}_{3}\right) \delta:$ 209.1, 204.2, 203.0, 149.0, 145.9, 136.0, 133.7, 122.6, 117.8, 112.7, 109.7, 70.4, 64.6, 63.9, 51.9, 46.7, $44.4,44.1,36.9,35.4,32.0,29.3,27.0,26.4,26.0,25.8$, 23.3, 22.5, 17.9, 17.9, 17.4. HRESIMS calcd for $\mathrm{C}_{31} \mathrm{H}_{47} \mathrm{O}_{3}$ $[\mathrm{M}+\mathrm{H}]^{+}$467.3525, found 467.3526.

\section{4 化合物 1 的合成}

在 $\mathrm{N}_{2}$ 氛围保护下, 取双环[3.3.1]壬烷-2,4,9-三酮 (5a) $(130 \mathrm{mg}, 0.28 \mathrm{mmol})$ 溶于 $6 \mathrm{~mL}$ 甲苯中，依次加入三 乙胺 $(0.233 \mathrm{mg}, 1.68 \mathrm{mmol}) 、 三$ 氯化钐 $(7 \mathrm{mg}, 0.028 \mathrm{mmol})$ 和 2,3-二乙酰氧基苯甲酰氰(19), 室温摚拌 $3 \mathrm{~h}$. 加水淬 灭后，乙酸乙酯萃取，旋干后不用过层析柱，加入 $6 \mathrm{~mL}$ 甲醇和碳酸钾 $(80 \mathrm{mg}, 0.57 \mathrm{mmol})$, 搅拌 $2 \mathrm{~h}$, 加水淬灭, 用乙酸乙酯和水萃取, 有机相加入无水硫酸镁干燥, 减 压分离得到化合物 1' (80 mg, 51\%). 采用同样的方法可 从 $5 b$ 得到 xanthochymol (1). 经多次尝试, 反应产率在 $51 \% \sim 55 \%$ 之间.

$(1 R, 5 R, 7 R)-3$-(3,4-二羟基苯甲酰基)-6,6-二甲基1-(5-甲基-2-异丙烯基-5-已烯)-5,7-二异戊烯基-2,4,9-三 酮-二环[3.3.1]壬烷(1'): ${ }^{1} \mathrm{H}$ NMR (600 MHz, $\left.\mathrm{CD}_{3} \mathrm{OD}\right) \delta$ : $7.25(\mathrm{~d}, J=2.1 \mathrm{~Hz}, 1 \mathrm{H}), 7.04(\mathrm{dd}, J=8.3,2.1 \mathrm{~Hz}, 1 \mathrm{H})$, $6.73(\mathrm{~d}, J=8.3 \mathrm{~Hz}, 1 \mathrm{H}), 5.05 \sim 4.97(\mathrm{~m}, 1 \mathrm{H}), 4.88(\mathrm{~s}, 1 \mathrm{H})$, $4.65(\mathrm{~s}, 1 \mathrm{H}), 4.63$ (brs, $1 \mathrm{H}), 4.62(\mathrm{~s}, 1 \mathrm{H}), 4.43(\mathrm{~s}, 1 \mathrm{H}), 2.70$ (dd, $J=13.2,9.4 \mathrm{~Hz}, 1 \mathrm{H}), 2.51(\mathrm{~d}, J=13.2 \mathrm{~Hz}, 1 \mathrm{H})$, $2.38 \sim 2.35(\mathrm{~m}, 1 \mathrm{H}), 2.30 \sim 2.22(\mathrm{~m}, 2 \mathrm{H}), 2.16 \sim 2.04(\mathrm{~m}$, $3 \mathrm{H}), 1.92 \sim 1.81(\mathrm{~m}, 2 \mathrm{H}), 1.76(\mathrm{~s}, 3 \mathrm{H}), 1.75(\mathrm{~s}, 3 \mathrm{H}), 1.73$ (dd, $J=13.9,4.0 \mathrm{~Hz}, 1 \mathrm{H}), 1.69$ (s, 3H), 1.69 (s, 3H), 1.68 (s, 3H), $1.55(\mathrm{~s}, 3 \mathrm{H}), 1.51 \sim 1.45(\mathrm{~m}, 3 \mathrm{H}), 1.15(\mathrm{~s}, 3 \mathrm{H})$, 0.99 (s, 3H); ${ }^{13} \mathrm{C}$ NMR $\left(151 \mathrm{MHz}, \mathrm{CD}_{3} \mathrm{OD}\right) \delta: 208.1$, 195.0, 195.0, 195.0, 151.6, 148.5, 145.9, 145.3, 134.4, $132.7,128.8,124.7,124.3,120.6,117.7,116.3,114.2$, $112.4,109.4,49.0,47.0,44.2,42.4,36.3,35.7,32.0,29.5$, $26.3,26.0,25.4,25.0,22.2,21.7,17.3,17.2,17.0$. HRESIMS calcd for $\mathrm{C}_{38} \mathrm{H}_{51} \mathrm{O}_{6}[\mathrm{M}+\mathrm{H}]^{+}$603.3686, found 603.3724 .

( $1 R, 5 R, 7 R$ )-3-(3,4-二羟基苯甲酰基)- 6,6 -二甲基1-(5-甲基-2-异丙烯基-5-已烯)-5,7-二异戊烯基-2,4,9-三 酮 -二环 [3.3.1] 壬烷 (xanthochymol，1): ${ }^{1} \mathrm{H}$ NMR (600 $\left.\mathrm{MHz}, \mathrm{CD}_{3} \mathrm{OD}\right) \delta: 7.21(\mathrm{~d}, J=2.1 \mathrm{~Hz}, 1 \mathrm{H}), 7.00(\mathrm{dd}, J=$ $8.3,2.1 \mathrm{~Hz}, 1 \mathrm{H}), 6.72(\mathrm{~d}, J=8.3 \mathrm{~Hz}, 1 \mathrm{H}), 5.08 \sim 5.03(\mathrm{~m}$, $1 \mathrm{H}), 4.90(\mathrm{~s}, 1 \mathrm{H}), 4.68 \sim 4.63(\mathrm{~m}, 2 \mathrm{H}), 4.53$ (br s, $2 \mathrm{H}$ ), $2.73(\mathrm{dd}, J=13.4,9.3 \mathrm{~Hz}, 1 \mathrm{H}), 2.61 \sim 2.55(\mathrm{~m}, 1 \mathrm{H})$, $2.61 \sim 2.55(\mathrm{~m}, 1 \mathrm{H}), 2.28(\mathrm{~d}, J=14.1 \mathrm{~Hz}, 1 \mathrm{H}), 2.16 \sim 2.10$ (m, $1 \mathrm{H}), 2.08 \sim 1.99(\mathrm{~m}, 3 \mathrm{H}), 1.97 \sim 1.88(\mathrm{~m}, 2 \mathrm{H}), 1.87 \sim$ $1.81(\mathrm{~m}, 1 \mathrm{H}), 1.75(\mathrm{~s}, 3 \mathrm{H}), 1.71(\mathrm{~s}, 3 \mathrm{H}), 1.71(\mathrm{~s}, 3 \mathrm{H}), 1.68$ (s, 3H), $1.63(\mathrm{~s}, 3 \mathrm{H}), 1.52(\mathrm{~s}, 3 \mathrm{H}), 1.53 \sim 1.48(\mathrm{~m}, 2 \mathrm{H})$, $1.45 \sim 1.41(\mathrm{~m}, 1 \mathrm{H}), 1.18(\mathrm{~s}, 3 \mathrm{H}), 1.02(\mathrm{~s}, 3 \mathrm{H}) ;{ }^{13} \mathrm{C} \mathrm{NMR}$ (151 MHz, CD $\left.{ }_{3} \mathrm{OD}\right) \delta: 209.7,194.8,194.6,194.6,151.6$, $148.0,146.0,145.3,134.9,132.7,128.6,124.6,124.2$, $120.4,117.0,116.4,114.1,112.6,109.5,68.8,60.6,49.3$, $47.0,43.8,42.8,36.7,35.8,31.8,29.4,26.4,26.1,25.5$, $25.0,22.2,21.8,17.4,17.2,16.8$. HRESIMS calcd for $\mathrm{C}_{38} \mathrm{H}_{51} \mathrm{O}_{6}[\mathrm{M}+\mathrm{H}]^{+} \quad 603.3686$, found 603.3730 . 
辅助材料(Supporting Information) 化合物 1, 1', 5a, $5 \mathbf{b}, \mathbf{6 a}, \mathbf{6 b}$ 的 ${ }^{1} \mathrm{H}$ NMR 和 ${ }^{13} \mathrm{C}$ NMR 的谱图. 这些材料可 以免费从本刊网站(http://sioc-journal.cn/)上下载.

\section{References}

[1] Ciochina, R.; Grossman, R. B. Chem. Rev. 2006, 106, 3963.

[2] Yang, X. W.; Grossman, R. B.; Xu, G. Chem. Rev. 2018, 118, 3508.

[3] Wu, S. B.; Long, C.; Kennelly, E. J. Nat. Prod. Rep. 2014, 31, 1158.

[4] Bridi, H.; Meirelles, G. C.; von Poser, G. L. Phytochemistry 2018, 155, 203.

[5] Gurevich, A. I.; Dobrynin, V. N.; Kolosov, M. N.; Popravko, S. A.; Riabova, I. D. Antibiotiki 1971, 16, 510.

[6] Grossman, R. B. Table of Naturally Occurring PPAPs. http:// http://www.uky.edu/ rbgros1/PPAPs (accessed 2021).

[7] Nicolaou, K. C.; Pfefferkorn, J. A.; Kim, S.; Wei, H. X. J. Am. Chem. Soc. 1999, 121, 4724.

[8] Spessard, S. J.; Stoltz, B. M. Org. Lett. 2002, 4, 1943.

[9] Usuda, H.; Kanai, M.; Shibasaki, M. Tetrahedron Lett. 2002, 43, 3621.

[10] Kraus, G. A.; Nguyen, T. H.; Jeon, I. Tetrahedron Lett. 2003, 44, 659.

[11] Mehta, G.; Bera, M. K. Tetrahedron Lett. 2004, 45, 1113.

[12] Kuramochi, A.; Usuda, H.; Yamatsugu, K.; Kanai, M.; Shibasaki, M. J. Am. Chem. Soc. 2005, 127, 14200.

[13] Karanjgoakar, C.; Rao, A. R.; Venkataraman, K.; Yemul, S.; Palmer,
K. Tetrahedron Lett. 1973, 14, 4977.

[14] Protiva, P.; Hopkins, M. E.; Baggett, S.; Yang, H.; Lipkin, M.; Holt, P. R.; Kennelly, E. J.; Bernard, W. I. Int. J. Cancer 2008, 123, 687.

[15] Saxe Einbond, L.; Mighty, J.; Kashiwazaki, R.; Figueroa, M.; Jalees, F.; Munoz Acuna, U.; LeGendre, O.; A. Foster, D.; J. Kennelly, E. Anticancer Agents Med. Chem. 2013, 13, 1540.

[16] Matsumoto, K.; Akao, Y.; Kobayashi, E.; Ito, T.; Ohguchi, K.; Tanaka, T.; Iinuma, M.; Nozawa, Y. Biol. Pharm. Bull. 2003, 26, 569.

[17] Iinuma, M.; Tosa, H.; Tanaka, T.; Kanamaru, S.; Asai, F.; Kobayashi, Y.; Miyauchi, K.; Shimano, R. Biol. Pharm. Bull. 1996, 19, 311 .

[18] Gustafson, K. R.; Blunt, J. W.; Munro, M. H. G.; Fuller, R. W.; McKee, T. C.; Cardellina, J. H.; McMahon, J. B.; Cragg, G. M.; Boyd, M. R. Tetrahedron 1992, 48, 10093.

[19] Baggett, S.; Mazzola, E. P.; Kennelly, E. J. Studies in Natural Products Chemistry, Vol. 32, Ed.: Atta-ur-Rahman, Elsevier, Amsterdam, 2005, p. 721.

[20] Wang, L.; Sun, L.; Wang, X.; Wu, R.; Zhou, H.; Zheng, C.; Xu, H. Org. Lett. 2019, 21, 8075.

[21] Wang, X.; Phang, Y.; Feng, J.; Liu, S.; Zhang, H.; Fu, W.; Zhou, H.; Xu, G.; Xu, H.; Zheng, C. Org. Lett. 2021, 23, 4203.

[22] Pepper, H. P.; Tulip, S. J.; Nakano, Y.; George, J. H. J. Org. Chem. 2014, 79, 2564.

[23] Wang, L.; Wang, X.; Zhang, G.; Fu, W.; Zhang, H.; Zhou, H.; Xu, H.; Zheng, C. Org. Chem. Front. 2021, 8, 2525. 